Published in final edited form as:

Science. 2015 September 18; 349(6254): 1282-1283. doi:10.1126/science.aad0584.

\title{
Strength in small numbers:
}

\section{A small-scale genome study of an indigenous population elucidates the genetics that influence height and weight}

\author{
Sarah Tishkoff \\ Departments of Genetics and Biology, Perelman School of Medicine, University of Pennsylvania, \\ Philadelphia, PA 19104, USA
}

Sarah Tishkoff: tishkoff@mail.med.upenn.edu

\begin{abstract}
Complex traits such as height and weight are influenced by multiple genes and by environmental factors. Because of this complexity, genetic studies of these traits often involve hundreds of thousands of individuals. These studies typically focus on urban populations, for which large sample sizes are readily available. For example, the GIANT consortium recently reported 697 genetic variants that explain $20 \%$ of the heritability of adult height (1), and 97 loci that account for just $2.7 \%$ of phenotypic variation in body mass index (BMI) (2). These discoveries required a staggering 250,000 to 300,000 individuals in total. And yet, on page 1343 of this issue, Fumagalli et al. report the identification of genetic variants influencing height and BMI based on data from just a few thousand Inuit individuals (3). These results exemplify how modern humans have adapted to diverse climates and diets.
\end{abstract}

The authors increase the statistical power for identifying genetic associations by first searching for targets of natural selection and then looking for association between those loci and potentially adaptive traits. Thus, they first identified genomic targets of natural selection in 191 individuals of Inuit ancestry residing in Greenland and then looked for an association of those regions with anthropometric and metabolic traits in a larger, but still modest, set of $\sim 4500$ Inuit individuals.

The Inuit population has inhabited the Arctic region for thousands of years, adapting to a cold climate and a diet rich in marine-derived fat. Using a single-nucleotide polymorphism (SNP) genotyping array designed to target metabolism-related genetic variants [the Metabochip (4)] and exome sequencing of a subset of 18 individuals, Fumagalli et al. identified several variable genome regions that differ in frequency in the Inuit population compared to Europeans and Chinese, possibly due to long-standing selective pressures to adapt to the Arctic environment. Genes found in these regions include those that code for fatty acid desaturases (FADS, important modulators of fatty acid composition), in addition to genes that play a role in fat distribution and in muscle and heart development. Derived variants that were targets of selection near the FADS loci were associated with smaller body size and shorter stature in the Inuit cohort. Two of these variants were modestly associated with a decrease in height in a cohort of 170,000 Europeans. These variants had not been significantly associated with height or body size in the larger European cohort, possibly because of their low frequency in that population. 
Fumagalli et al.'s study shows that inclusion of ethnically diverse populations that have adapted to extreme environments for thousands of years may be particularly informative for genetic studies of variable traits. The analysis of genetic variants in individuals with phenotypes at the extremes of the trait distribution has been successfully used to identify functionally relevant genetic variants within populations. For example, analysis of European-descent individuals with extremely low concentrations of low-density lipoprotein (LDL) cholesterol led to the identification of loss-of-function mutations in the gene coding for PCSK9, a key factor for lowering cholesterol (5) that is now the target of novel drug treatments (6).

Adaptation to diverse environments during human evolution has resulted in populations with phenotypes that are at the extremes of the trait distribution across all humans. Extreme phenotypes resulting from natural selection may in some cases be due to relatively few genetic variants with large effect. Integration of scans for natural selection, to narrow down candidate loci, with phenotype association studies can be particularly informative for identifying genetic variants associated with complex traits, even in studies with small sample sizes.

For example, this approach has been used to identify genetic loci influencing the extreme short stature trait in Central African huntergatherer populations, a trait thought to be an adaptation to a tropical environment $(7,8)$. It has also been used to find loci influencing physiologic adaptation to high altitude in Tibet, South America, and Ethiopia (9). These studies show that smaller-scale studies of ethnically diverse populations living in a range of different environments and experiencing local adaptation are important and complementary to large genome-wide association studies (GWAS) of urban populations (see the figure).

The study of indigenous populations can be informative for several reasons. For example, although environments may vary considerably between populations, the environments within individual populations are often relatively homogeneous (similar diets, cultural habits, and environmental exposures). This can improve the power to identify genetic factors influencing complex phenotypes. In addition, increased levels of inbreeding in some small indigenous populations lead to regions of the genome that are identical within and among individuals and that can be useful for identifying rare variants influencing complex traits (10).

More fundamentally, investigation of such populations is crucial to further distribute the benefits of "precision medicine" and the associated development of targeted therapeutics. However, indigenous populations, particularly those living in rural areas with little access to health care, can be very challenging to study. Great care must be taken to avoid coercion and to be respectful of local practices, customs, and beliefs. This issue has been of particular concern to some Native American and Australian Aboriginal populations who may not want to participate in genomics research because of cultural beliefs. Some indigenous populations are also wary of genomics research owing to concerns about exploitation. There is valid concern about commercialization of information obtained from studies of indigenous populations. However, that concern must be balanced by the important need to develop therapeutics that will benefit all populations, not just those in developed countries. Thus, 
dialogue between researchers and indigenous communities is needed to ensure benefit sharing. It is critical to include diverse peoples in genomic studies to understand the phenotypic impact of the full range of human genetic variation and to ensure that all peoples benefit from this knowledge.

\section{References}

1. Wood AR, et al. Nat Genet. 2014; 46:1173. [PubMed: 25282103]

2. Locke AE, et al. Nature. 2015; 518:197. [PubMed: 25673413]

3. Fumagalli M, et al. Science. 2015; 349:1343. [PubMed: 26383953]

4. Voight BF, et al. PLOS Genet. 2012; 8:e1002793. [PubMed: 22876189]

5. Cohen JC, Hobbs HH. Science. 2013; 340:689. [PubMed: 23661745]

6. Sheridan C. Nat Biotechnol. 2015; 33:785. [PubMed: 26252118]

7. Jarvis JP, et al. PLOS Genet. 2012; 8:e1002641. [PubMed: 22570615]

8. Perry GH, et al. Proc Natl Acad Sci USA. 2014; 111:E3596. [PubMed: 25136101]

9. Scheinfeldt LB, Tishkoff SA. Genome Biol. 2010; 11:133. [PubMed: 20979669]

10. Browning SR, Thompson EA. Genetics. 2012; 190:1521. [PubMed: 22267498] 

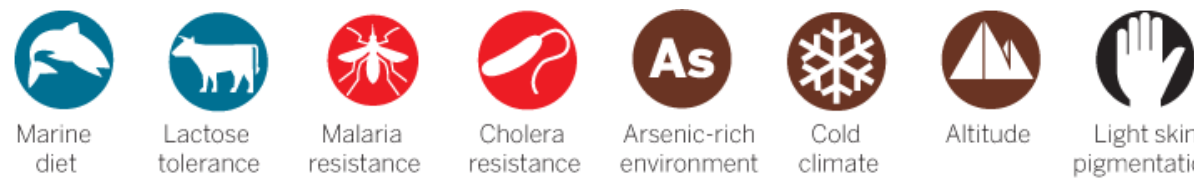

Light skin pigmentation

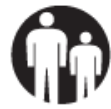

Short stature

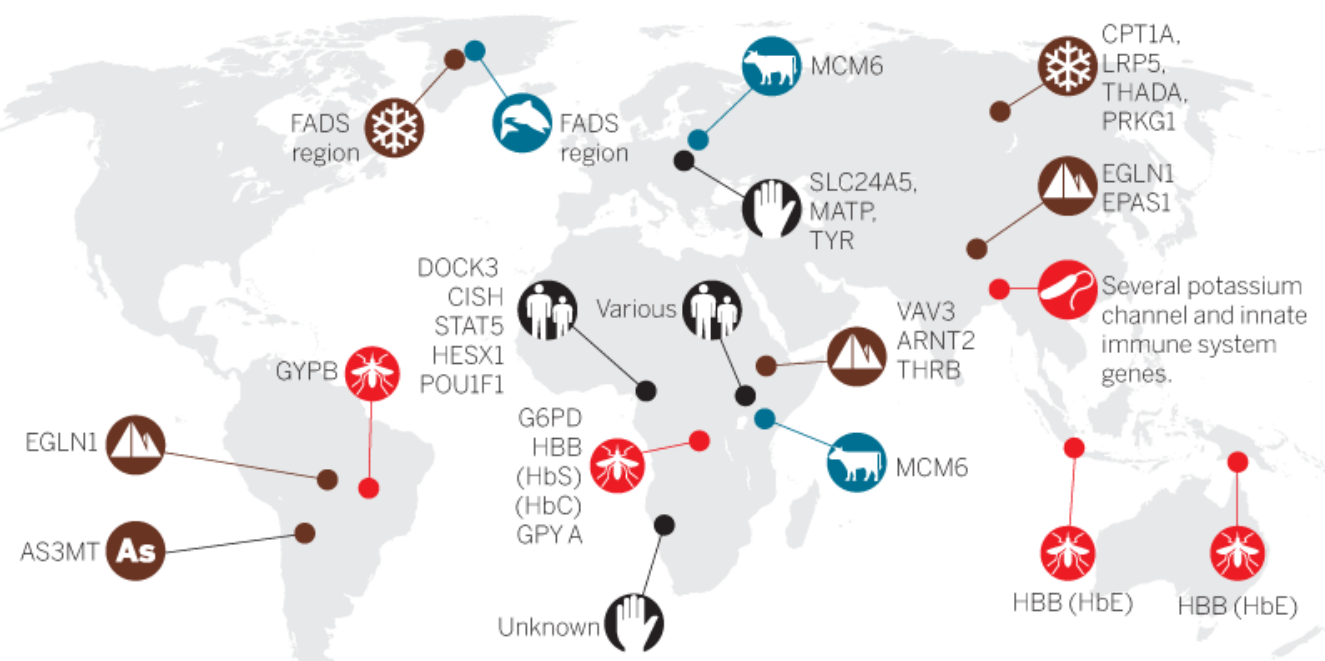

Global distribution of locally adaptive traits

Adaptation to diverse environments during human evolution has resulted in phenotypes that are at the extremes of the global distribution. Fumagalli et al. have integrated scans of natural selection and GWAS to identify genetic loci associated with adaptation to an Arctic environment. 